UAH Final Report

for

NASA Grant No. NAG8-159

\title{
ENDOSCOPIC INSPECTION USING A PANORAMIC ANNULAR LENS
}
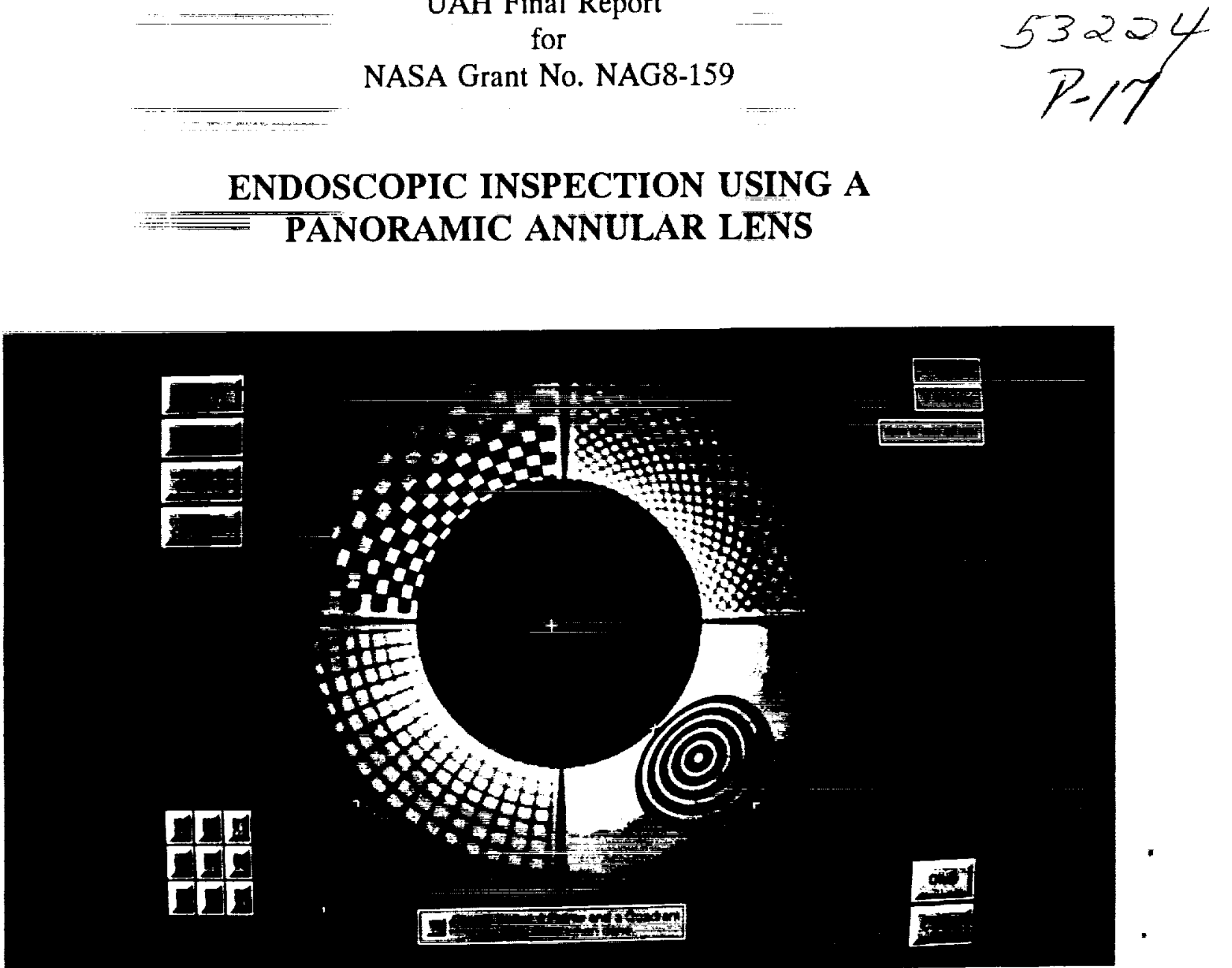

Submitted to

George C. Marshall Space Flight Center

National Aeronautics and Space Administration

Marshall Space Flight Center

Huntsville, Alabama 35812

\section{Prepared by}

John A. Gilbert, Ph.D.

Department of Mechanical Engineering

University of Alabama in Huntsville

Huntsville, Alabama 35899

(205) 895-6029
Donald R. Matthys, Ph.D.

Physics Department

Marquette University

Milwaukee, Wisconsin 53233

(414) 288-1494

December, 1991 
UAH Final Report

for

NASA Grant No. NAG8-159

\title{
ENDOSCOPIC INSPECTION USING A PANORAMIC ANNULAR LENS
}

\author{
Submitted to \\ George C. Marshall Space Flight Center \\ National Aeronautics and Space Administration \\ Marshall Space Flight Center \\ Huntsville, Alabama 35812
}

Prepared by

John A. Gilbert, Ph.D.

Department of Mechanical Engineering

University of Alabama in Huntsville

Huntsville, Alabama 35899

(205) 895-6029
Donald R. Matthys, Ph.D.

Physics Department

Marquette University

Milwaukee, Wisconsin 53233

(414) 288-1494

December, 1991 


\section{TABLE OF CONTENTS}

Description $\quad \underline{\text { Page }}$

$\begin{array}{lr}\text { Cover Page } & 1\end{array}$

Table of Contents $\quad 2$

1. Purpose of Research Effort

2. Research Scheduled During the Performance Period 3

3. Work Accomplished During the Performance Period 3

a) Characterization of the panoramic imaging system 3

b) Prototype system for inspection and image acquisition 5

c) Image processing $\quad 6$

$\begin{array}{lr}\text { d) Prototype demonstration } & 8\end{array}$

4. Potential Applications for Cavity Inspection $\quad 10$

5. Potential Applications for Panoramic Imaging 12

$\begin{array}{lr}\text { 6. Personnel } & 14\end{array}$

$\begin{array}{ll}\text { 7. Publications and Presentations } & 14\end{array}$

$\begin{array}{lr}\text { 8. Conclusions } & 15\end{array}$

$\begin{array}{lr}\text { 9. Acknowledgements } & 16\end{array}$ 


\title{
UAH Final Report \\ for \\ NASA Grant No. NAG8-159 \\ ENDOSCOPIC INSPECTION USING A PANORAMIC ANNULAR LENS
}

\author{
prepared by \\ John A. Gilbert, Ph.D. \\ Department of Mechanical Engineering \\ University of Alabama in Huntsville \\ Donald R. Matthys, Ph.D. \\ Physics Department \\ Marquette University
}

\section{Purpose of Research Effort}

The objective of this one year study was to design, build, and demonstrate a prototype system for cavity inspection. A cylindrical view of the cavity interior was captured in real time through a compound lens system consisting of a unique panoramic annular lens and a collector lens. Images, acquired with a digitizing camera and stored in a desktop computer, were manipulated using image processing software to aid in visual inspection and qualitative analysis.

\section{Research Scheduled During the Performance Period}

The tasks to be completed were to a) characterize the panoramic imaging system, b) design and build a prototype system for inspecting cavities and acquiring images, c) develop techniques for processing panoramic images, and d) demonstrate a prototype system for cavity inspection.

\section{Work Accomplished During the Performance Period}

a) Characterization of the panoramic imaging system:

The panoramic annular lens (PAL) consists of a single piece of glass with spherical surfaces that produces a flat annular image of the entire 360 degree surround of the optical axis of the lens. A virtual annular-shaped image of the surroundings of the lens is formed inside the lens; this virtual image can be imaged onto a film or sensor by the use of a transfer lens. Figure 1 shows the geometric structure of the PAL. The thick lines indicate reflecting surfaces.

If a cavity can be regarded as a cylindrical rather than a spherical volume, the image information can be transformed, using stretching methods, onto a flat surface creating a 2-D representation of the 3-D cylindrical surface. This phenomenon called Flat Cylinder Perspective (FCP), forms the basis for the image produced by a panoramic annular lens. In an FCP mapping all parallel rays are focused to a single point, unlike the traditional perspective technique in which parallel 


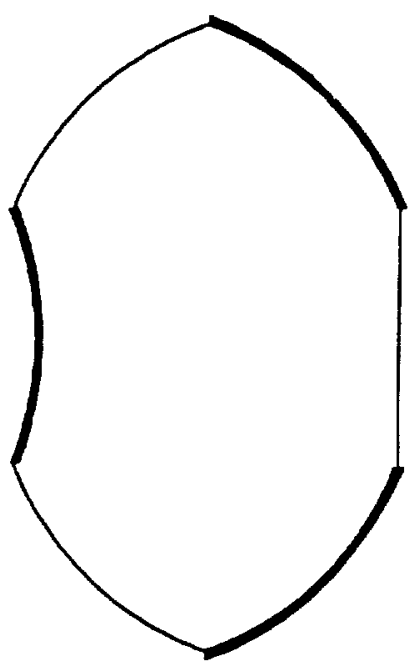

Figure 1. Physical shape of a panoramic annular lens (PAL).
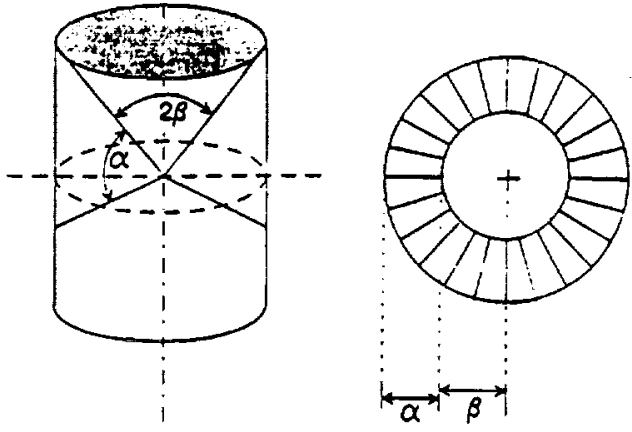

Figure 2. The PAL forms an image using flat cylinder perspective.

lines with different directions are focused to different points on a line (the horizon). Figure 2 shows a diagram representing flat cylinder perspective and shows the limiting angles involved in determining the field of view. Obviously, it would be desirable to make $\alpha$ large and $\beta$ small, so as to maximize the viewed surface, but restrictions are imposed on the values of these parameters by the limited range of values of the index of refraction that can be obtained in commercially available glasses. The width of the annular FCP image corresponds to the size of the acceptance angle $\alpha$, and each concentric ring in the image plane is the locus of points recorded at a fixed angle to the optical axis. The lens produces an annular FCP image where the width of the annular image corresponds to the vertical viewing angle, and where each concentric ring in the image plane is the loci of points recorded at a constant horizontal field angle. A ray diagram showing how the rays from the cavity wall traverse the lens and form

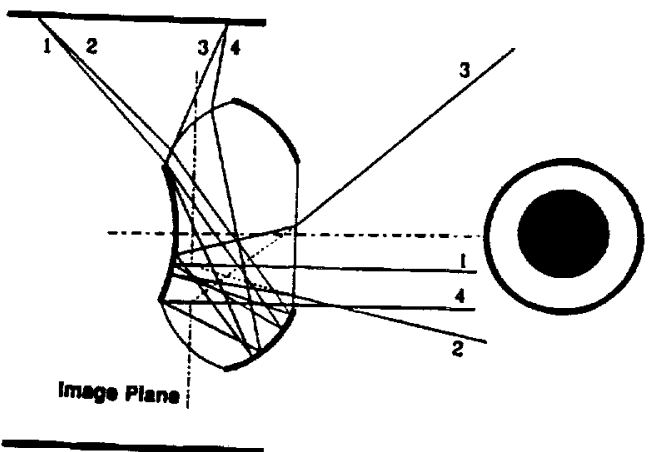

Figure 3. Ray diagram for a panoramic annular lens. a virtual image inside the PAL itself is shown in Figure 3. Figure 4 shows the use of a transfer lens to image the internal virtual annular-shaped image onto an external film or sensor.

A PAL prototype lens system was provided for use on the project by Optechnology, Inc. The system included a $38 \mathrm{~mm}$-diameter PAL, and an $\mathrm{f} / 1.4,25 \mathrm{~mm}$ focal length collector lens. The 
optics were packaged with a standard " $C$ " mount so that the system could be attached to a standard video camera. Figure 5 shows a schematic of the PAL system.

Initial evaluation of the prototype system showed that it had a field of view which extended from about -20 degrees behind the lens to about 25 degrees in front of the lens $\left(\alpha=45^{\circ} ; \beta=65^{\circ}\right)$. A commercial lens design program (OPTEC-II) was acquired to analyze the optical properties of the PAL and its associated collector lens, and tests were performed to study the aberration in the PAL based on ray tracing and the method of caustics. The results of this study showed that design changes could be made in the PAL system to increase the clarity and resolution of the PAL image. Application of the lens design program is currently helping to optimize the performance of other PAL prototypes.

b) Prototype system for inspection and image acquisition:

Even though many of the optical characteristics of the PAL and collector lens were evaluated, an optical system for visual observation and measurement requires more than a lens. The problems of illumination, image acquisition, and data storage had to be addressed.

Figure 6 shows the PAL system adapted for visual inspection. In this example, the lens system is surrounded by a ring containing a number of discrete light sources for illumination within a cavity. The PAL prototype is mounted on a standard CCD camera (Pulnix TM-845) having a resolution of $512 \mathrm{x}$ 512 pixels. The hardware platform selected for acquiring and storing images was an 80386 microprocessor running on a standard AT bus under the MS-DOS operating system (Dell System 310). Images are acquired by a standard commercial frame grabber and processor (Matrox MVP. AT) and stored in the Dell 310 computer.

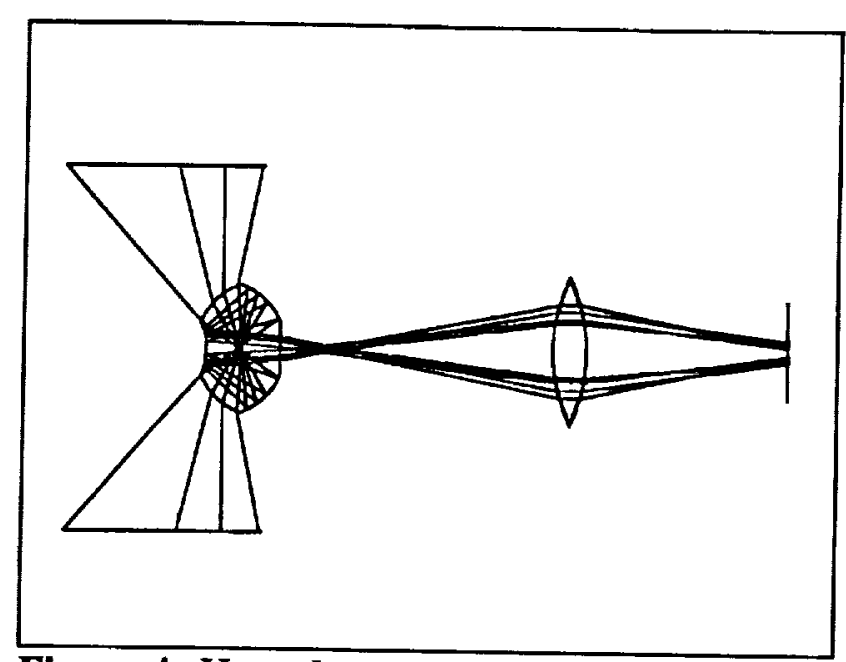

Figure 4. Use of a transfer lens to produce a real annular image.

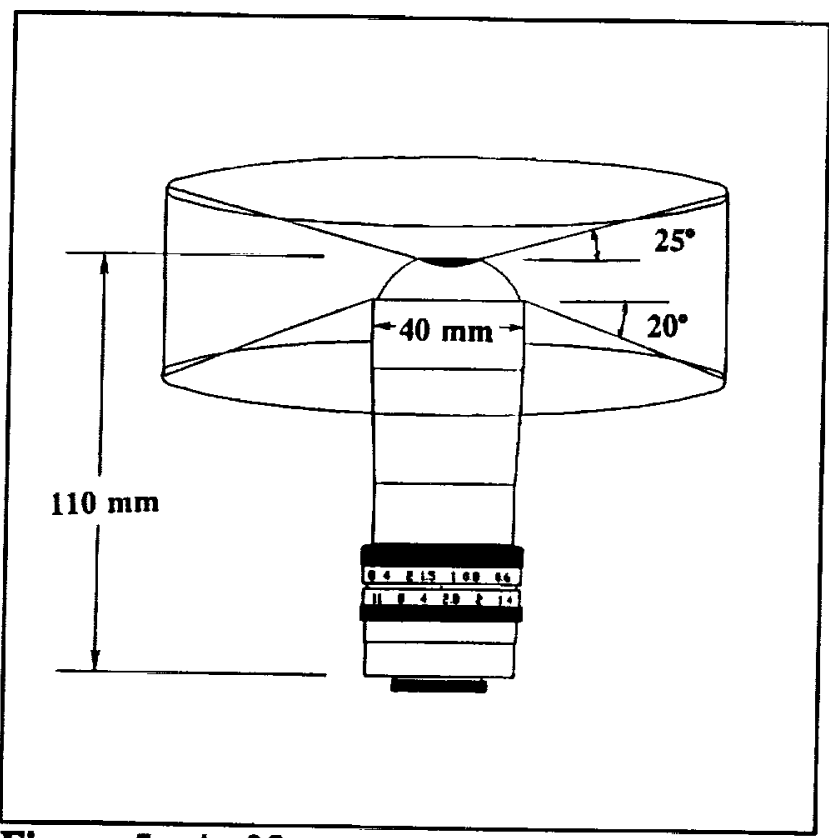
system provided to the project by Optechnology, Inc.
Figure 5. A $38 \mathrm{~mm}$ diameter PAL imaging 


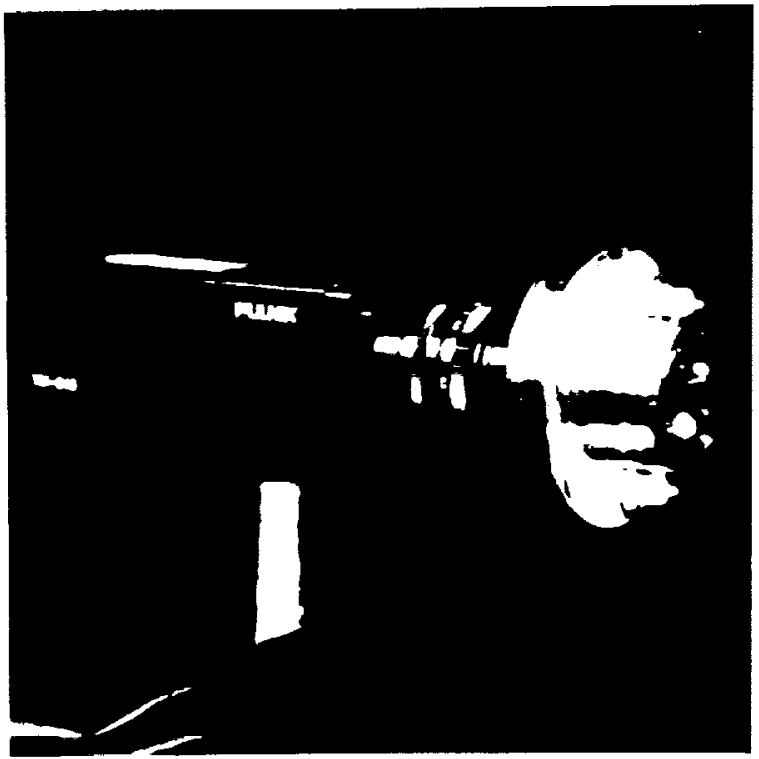

Figure 6. A ring of lights may be used to surround a PAL system for illumination within cavities.

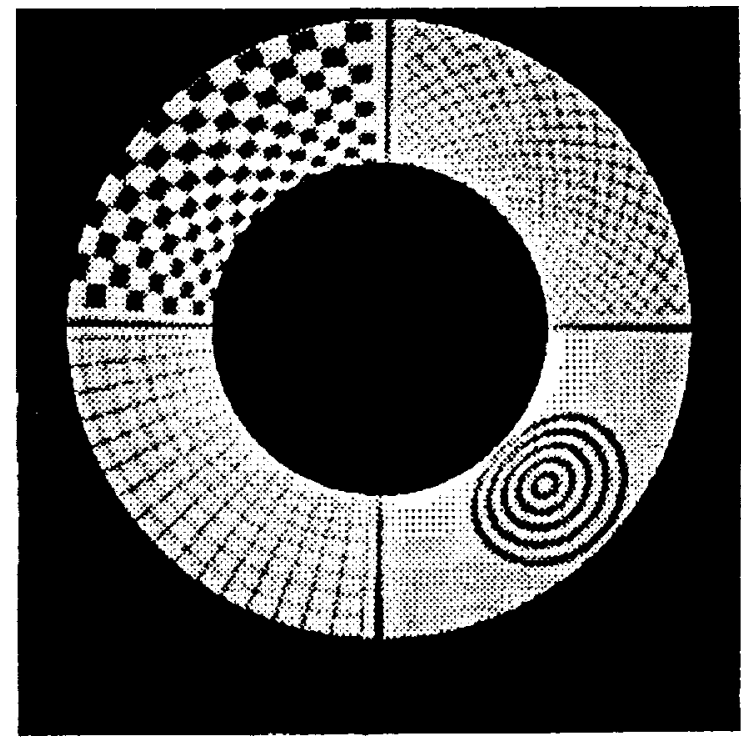

Figure 7. The image obtained when a PAL is positioned along the axis of a cylindrical pipe, the interior of which is covered with a test pattern.

The 386-class machine has a clock speed comparable to a main frame, yet it is designed to operate in the work area without the need for specially controlled operating environments, it is relatively inexpensive, mass storage is very cheap, parts are readily available world-wide, and the bus architecture allows for integration and intelligent control of user-supplied boards. Digital image storage permits signal-to-noise enhancement algorithms to be applied to eliminate, or at least greatly reduce, undesirable signal contaminants. The computer's archival capability facilitates further analyses by several individuals and by different software algorithms, without the need for repeating the inspection. Images and their salient features can be readily compared to data recorded in other inspections, automated data analysis is possible, and independent third party evaluations can be made without relying on the expertise or subjective evaluation of a single investigator.

Figure 7 illustrates a number of these attributes. It shows a reconstruction of the digital image acquired and stored when the prototype, shown in Figure 5, was positioned along the axis of a cylindrical pipe with a test drawing placed around its interior surface.

c) Image processing:

Direct visual interpretation of a PAL image is sometimes confusing for the unskilled observer. With this in mind, an algorithm was developed to allow the annular shaped images to be linearized for viewing and measurement purposes. It must be recognized that there is no easy way to present a nonrectangular image in a rectangular format without distortion. This is 
essentially the same problem as making a flat map out of a round globe. However, the type of distortion introduced can be chosen and controlled by the choice of mapping scheme that is used (equal maximal dimensions, equal areas, etc.). The mapping used here maintains equal maximal dimensions by 'rolling' the annular image along its outer circumference and moving all the pixels between the contact point and the center of the image to a vertical line in the final rectangular image.

Figure 7 was used to test the linearization routines. The test drawing contains a different pattern, i.e., diamonds, squares, checkerboard, and concentric circles, in each quadrant of the cavity wall. Referring to Figure 7, it can be seen that the distortion introduced by the FCP mapping is not severe, and the image is clearly recognizable. However, if it is desired to 'straighten out' the image, two stages of linearization are needed: (1) tangential linearization and (2) radial linearization. In the tangential linearization, a wedge-shaped portion of the annular image of the inside of the pipe is converted into a rectangular section. Next, because the annular image is not linear in the radial direction, a vertical stretching of the rectangular image is required; this second process is radial linearization.

The first step in linearizing the images obtained from the panoramic annular lens is to specify the size of the image. This is done by entering four $(x, y)$ locations into the computer. The first three points are chosen on the outer circumference of the image. This allows the computer to calculate the outer radius and the center of the image. The last point is chosen anywhere along the inner circumference and allows the machine to determine the inner radius and the image height. An interface was designed to allow a user to select one of eight different quadrants (1-4, north, south, east, or west) of the image to be linearized. Once the points and the quadrant are chosen, the machine knows the entire segment that is desired and can proceed to straighten it out.

First the center location of the annulus is calculated, and then the height and width of the output rectangular image are determined in units of pixels. The final width of the image will be equal to the length in pixels of the outer circumference of the selected quadrant. Figure 8 illustrates how samples (shown as 'o's) in the annular image are mapped into a rectangular array. After the outer radial length is selected at one end of the specified quadrant, the radial angle is incremented until the other end of the specified region is reached; then the radius is

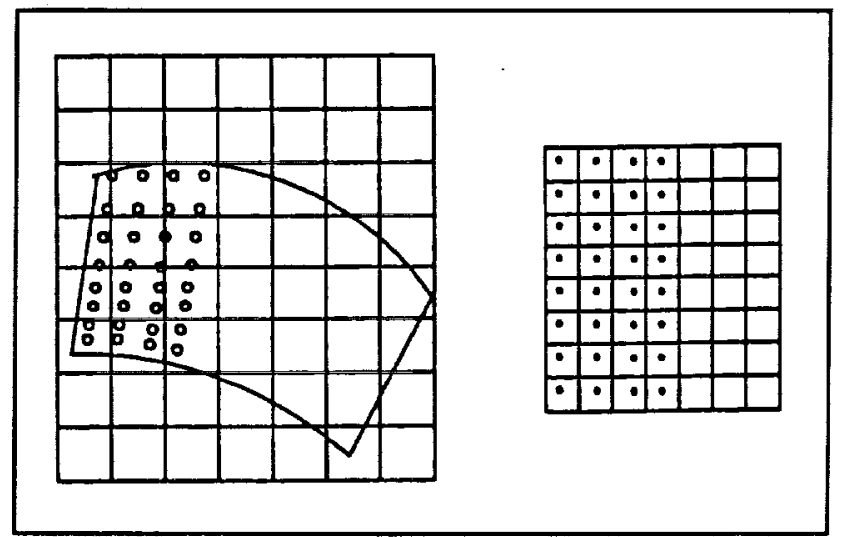

Figure 8. Mapping polar into cartesian coordinates. decremented and the process is repeated. After this linearization, the number of columns in the image, which is determined by the angular increment used, is adjusted, by averaging, to have the same number as the outer arc length of the selected quadrant. Next the vertical height of the rectangle is then determined by calibrating the system. This calibration was accomplished for the $38 \mathrm{~mm}$ diameter PAL system by covering the 
interior cavity wall with a grid. Since the vertical and horizontal lines of the grid have the same spacing on the walls of the cavity, they should be equally spaced on the final linearized image. The spacing between vertical lines is taken as a reference and the spacing of horizontal lines in the rectangular image obtained after linearization is adjusted to obtain such a separation.

Since a portion of the source image is being magnified by performing this linearization, a one-toone mapping of source to destination pixels is not guaranteed. When an image is magnified, one pixel in the source image may be mapped to many pixels in the destination image. This source to destination pixel mapping is done from the perspective of the destination image. This reverse mapping is required to guarantee that every pixel in the destination image is given a value. Without the one-to-one correspondence between source and destination pixels it cannot be guaranteed that some source pixel will be mapped into each and every destination pixel. If there were no reverse mapping then there would be pixels that are not given a pixel value. These voids would degrade the appearance of the destination image.

Reverse mapping traverses the destination image space a pixel at a time and uses the transformation function to determine which pixel of the source image would be involved in producing the destination pixel.

However, reverse pixel mapping creates a second problem. There will be fractional pixel addresses. These occur when the source image pixel that contributes to a destination pixel's value is calculated. To deal with these fractional addresses an interpolation is performed to calculate a new value for some point with a non-integer $(x, y)$ location that is situated between other points of known value. The comer points of known value that surround the point being calculated ('P', in our case) have a larger impact on the value of the calculated point than points further away. Assuming that the points are close together and that the intensity does not change rapidly with position allows the use of simple linear interpolation.

In Figure 9, a pixel has a non-integer address. The intensity of the pixel [P] with non-integral address values of $(x, y)$ is derived from the intensities of pixels [A], [B], [C], and [D] according to their relative distances from the calculated address of the transformed pixel. A twodimensional interpolation is performed by making three linear interpolations of intensity values as shown in Figure 10. The grey scale values of $[A]$ and $[B]$ are interpolated first, and then the gray values of $[C]$ and $[D]$. Using these two interpolated values as endpoints, the final result to be used as the grey scale value is interpolated linearly in the vertical direction. Figure 11 shows the final image obtained after applying both tangential and radial linearizations to the fourth quadrant of the image shown in Figure 7.

d) prototype demonstration:

Figure 12 shows a photograph of a PAL probe demonstrated to NASA personnel on November 18, 1991. For this purpose, the system in Figure 6 was mounted within a 3" diameter aluminum housing; an acrylic window was used to surround the optics for viewing and protection. The power supply, shown in the upper portion of Figure 12, was used to control the intensity provided 


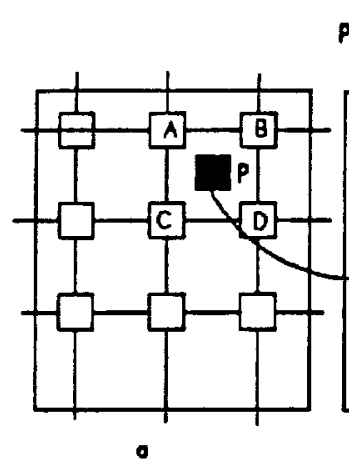

vouree Image
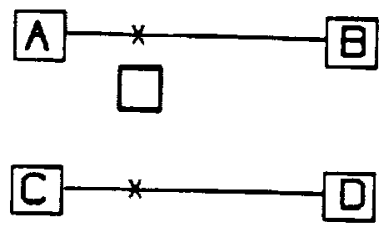

a.) Horizontal Inferpolation

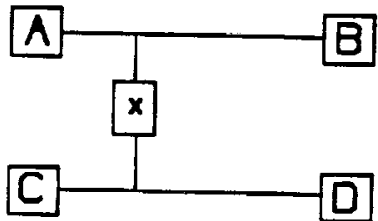

b.) Vertical Interpolation
Figure 9. Reverse mapping from final image to source image.
Figure 10. Interpolation of intensity values for non-integer coordinates.

by the illuminating ring.

During the demonstration, the prototype was lowered into a pipe having an inner diameter of

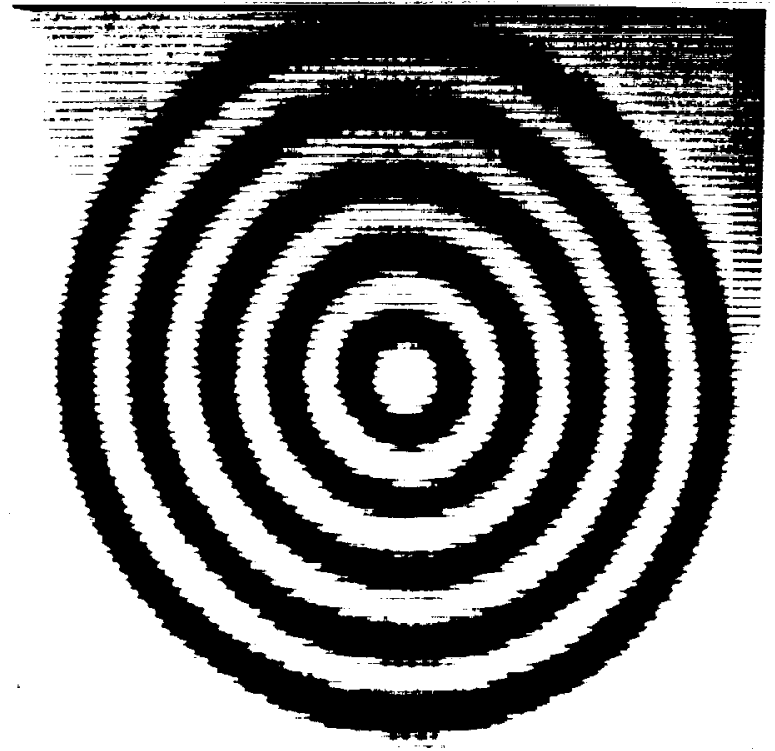

Figure 11. Linearized image of one quadrant of the test pattern.

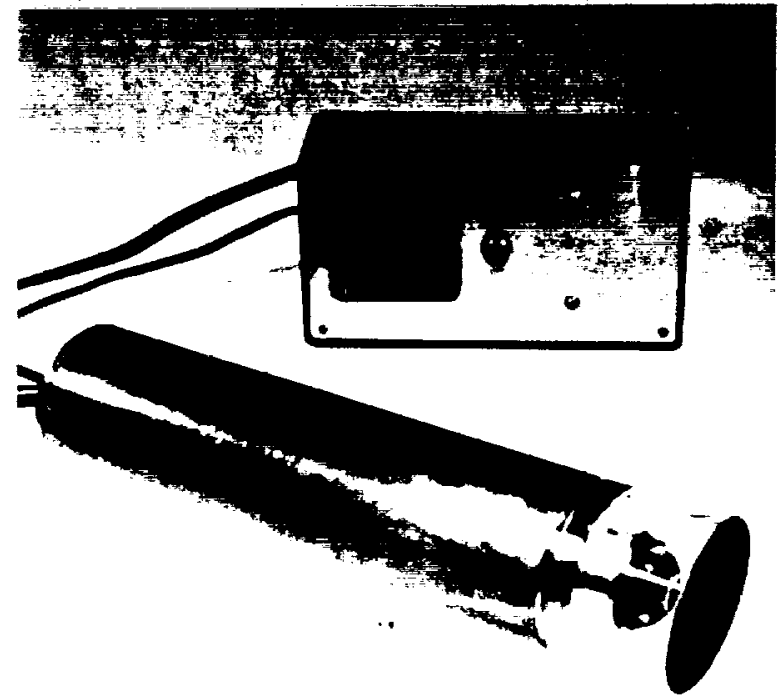

Figure 12. A 3" diameter PAL probe demonstrated to NASA/MSFC. 
3.875". A number of different test patterns, attached to the inner wall of the pipe, were viewed on a monitor. A test pattern, similar to that shown in Figure 7, was stored in the computer to demonstrate image processing and linearization.

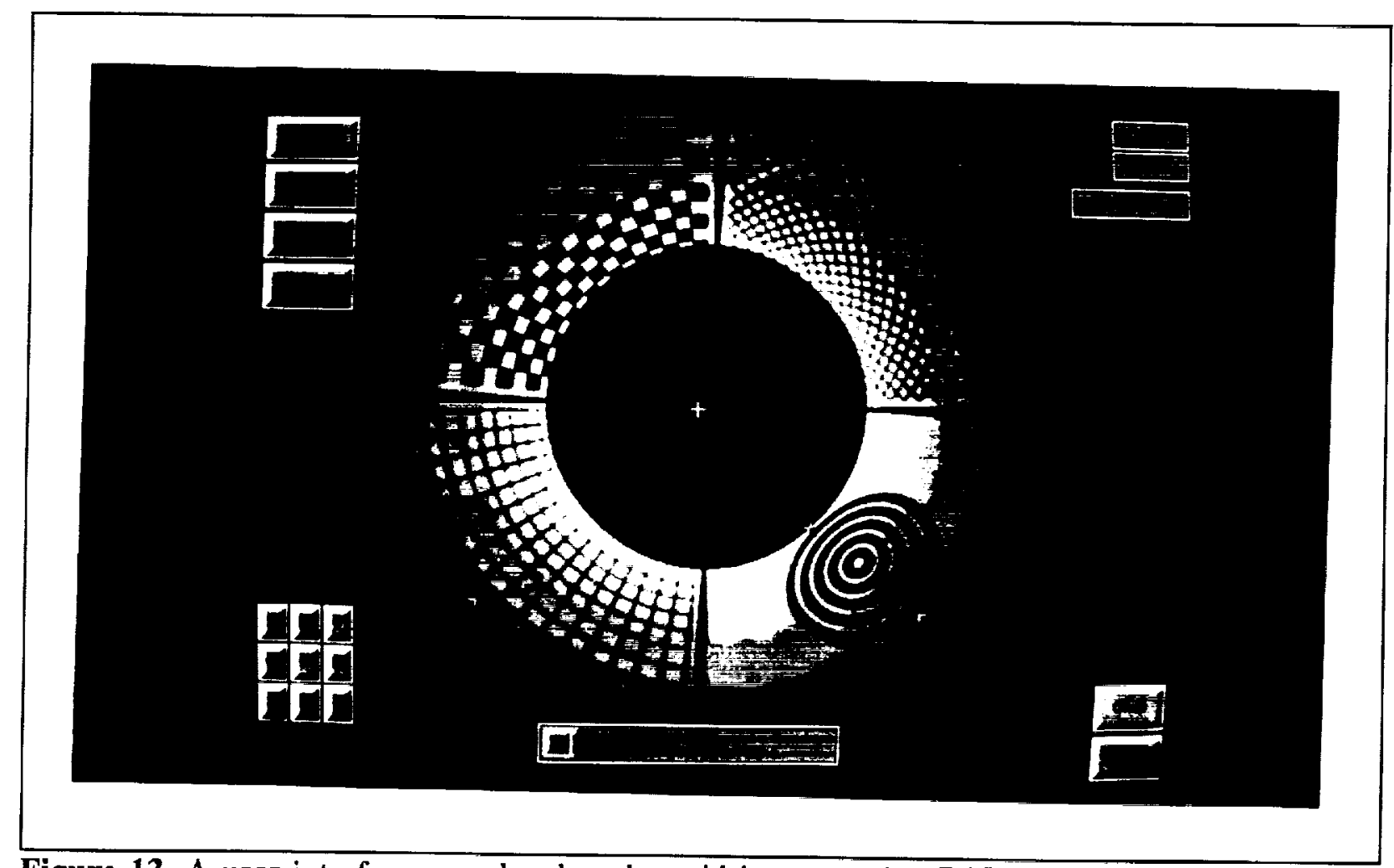

Figure 13. A user interface was developed to aid in processing PAL images.

The graphical user interface, illustrated in Figure 13, was designed and developed by students working on the project. It is based on an extensive library of graphical tricks, developed and published by MIT, and includes buttons, scroll boxes, message boxes, and text keyins. This mouse driven software package will be included in a PAL measurement system scheduled for delivery to NASA's Marshall Space Flight Center in June, 1992.

\section{Potential Applications for Cavity Inspection}

Potential applications of the inspection system include detection of surface and near-surface cracks in weldments, detection of seams and foldovers in castings, monitoring of wear, and detection of structural failures. These applications are typically encountered in aerospace structures and propulsion systems where many components, designed to function at high temperatures and pressures, must be periodically inspected to avoid catastrophic failures.

For example, a visual inspection of the Main Combustion Chamber (MCC) throat of the Space Shuttle Main Engine (SSME) is required during manufacture, after each test firing, and as part of refurbishment between missions. The temperature is hottest in this region and periodic 


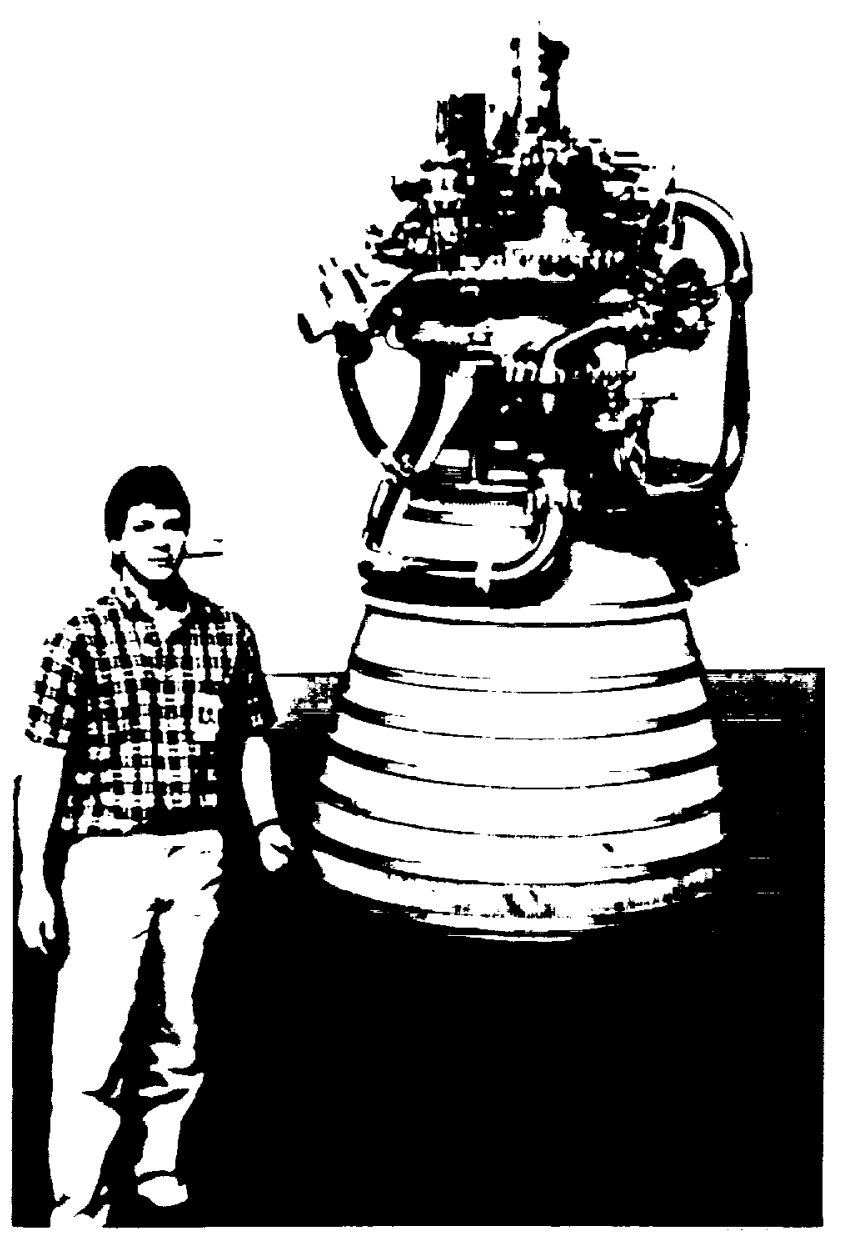

Figure 14. Photograph of an RL10 rocket engine. inspections are performed in an attempt to detect crack initiation, to monitor crack growth, and to observe surface roughness. Current inspection techniques require an inspector to insert their head into the throat and visually examine the nozzle contour. Cracks must be at least 3.0 inches or longer before they are recorded on a map of the MCC. The application of the PAL system should allow smaller cracks to be detected and, at the same time, eliminate the hazards associated with using support personnel to physically climb up into the nozzle. Moreover, the PAL system may eliminate the need for disassembling systems for inspection of their internal components; thereby, saving time and money. The PAL system may also find practical application in the Space Exploration Initiative. The Earth to Mars propulsion system is projected to consist of space-based engines with the same thrust class as the J-2. After Earth to Orbit delivery of the propulsion system, the PAL system could be used for damage assessment of the large expansion ratio nozzle and the chamber. Both the SSME and the J-2 have been targeted for future work.

Some steps have already been taken to inspect rocket engines; Figure 14, for example, is a photograph of an RL10 engine. Built by Pratt \& Whitney, the RL10 has earned its reputation as the United States' most reliable upper-stage engine and has been responsible for launching numerous satellites and spaceprobes on a variety of earth orbital and interplanetary missions. The current engine model, the RL10A-3-3A, powers the Centaur stage of the USAF's newest Expendable Launch Vehicle, the Titan IV. This system will be used to launch a number of heavy payloads for the Department of Defense during the 1990s. Figure 15 shows the panoramic image taken through a PAL system for visual inspection inside the nozzle throat.

Smaller PAL prototypes are currently being developed for use in medical applications to visually inspect internal organs. Figure 16, for example, shows that a $6.3 \mathrm{~mm}$ diameter PAL can be attached to the distal end of a cystoscope. These smaller systems should find widespread applications for nondestructive evaluation of aerospace components. In addition to those mentioned earlier, many of the critical components of the SSME are located in regions that are difficult to reach and inspect. A typical example is the LOX heat exchanger, which contains four tubing welds. Flaws may result from lack of penetration in the original weld, and cracks may 


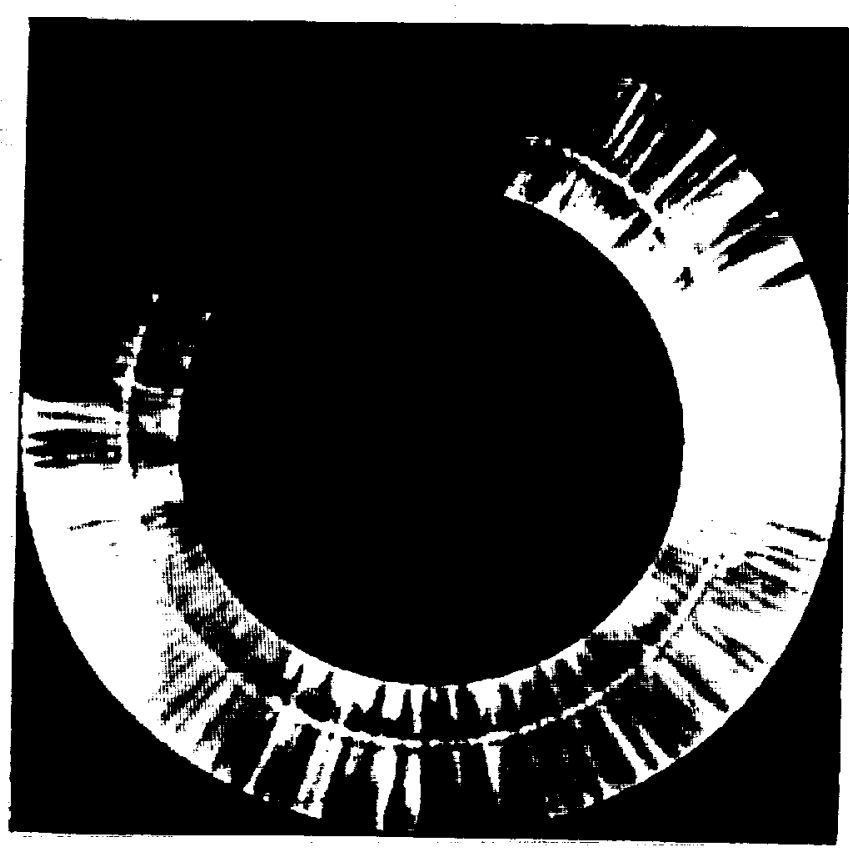

Figure 15. A PAL photograph taken inside the nozzle of an RL10.

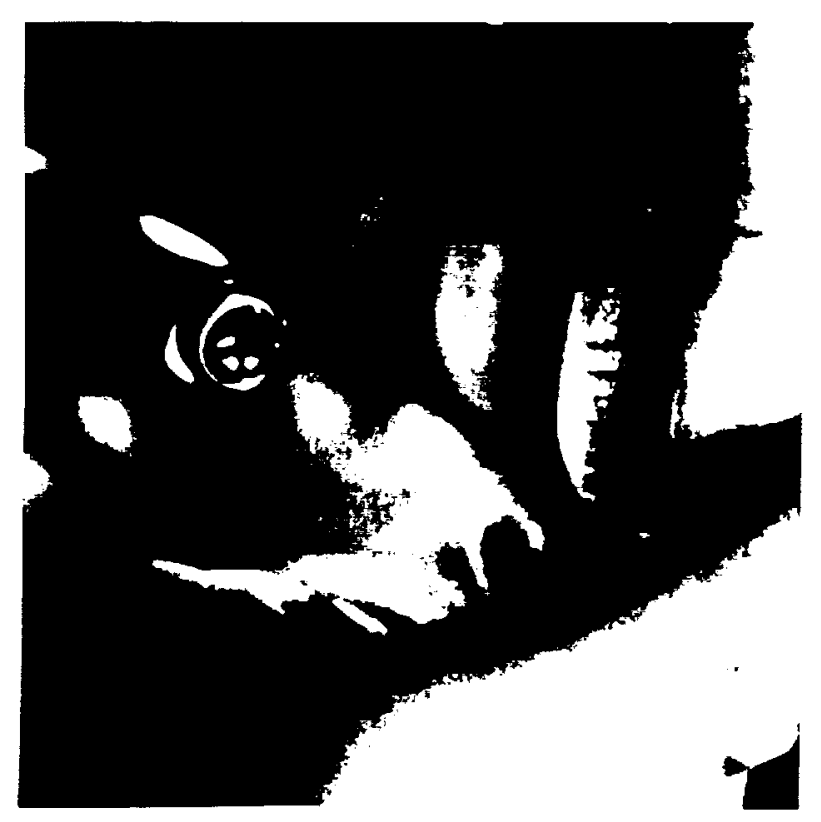

Figure 16. A $6.3 \mathrm{~mm}$-diameter prototype is currently under development.

be induced in these locations by forming, or by low cycle fatigue during service. The most difficult weld to access is located approximately 30.6 in. $(0.78 \mathrm{~m})$ from the inlet, through tubing 0.190 in. $(4.83 \mathrm{~mm}) \mathrm{ID}$, past a 90 degree, $0.69 \mathrm{in} .(18 \mathrm{~mm})$ radius bend, and approximately halfway around a loop $16.5 \mathrm{in} .(42 \mathrm{~cm})$ diameter. Efforts to miniaturize the PAL and adapt it to a flexible boroscope may ultimately lead to the development of a practical working instrument for inspecting these components in the field through existing ports with minimal inspection effort.

\section{Potential Applications for Panoramic Imaging}

In addition to ground based monitoring and performance evaluations of aerospace components, PAL systems are being targeted for use in the space operating environment. Figure 17 illustrates a preliminary design for a panoramic imaging system called SEASIS. The imaging system will be contained in an endmass to be deployed via NASA's Small Expendable Deployer System (SEDS) from the second stage of a Delta II launch vehicle via a $40 \mathrm{~km}$-long tether. The panoramic images recorded during the mission will be incorporated with data gathered from a three-axis accelerometer to study tether dynamics. This information will be valuable, since tethered systems will be used in many space based applications including the construction and use of relatively large tethered platforms. Figure 18, for example, shows a configuration which utilizes Shuttle external tanks in a raft format to form a structure in space. In this example, tethers are used as structural elements in an evolving Space Station and for links (power, data transfer, etc.) between different platforms designed for various science and applications purposes. Some of the platforms would take advantage of the facilities of the station for maintenance and repair while being isolated from contamination and mechanical disturbances. Others could be used to facilitate storage of liquid propellants and dangerous fluids, to provide a 


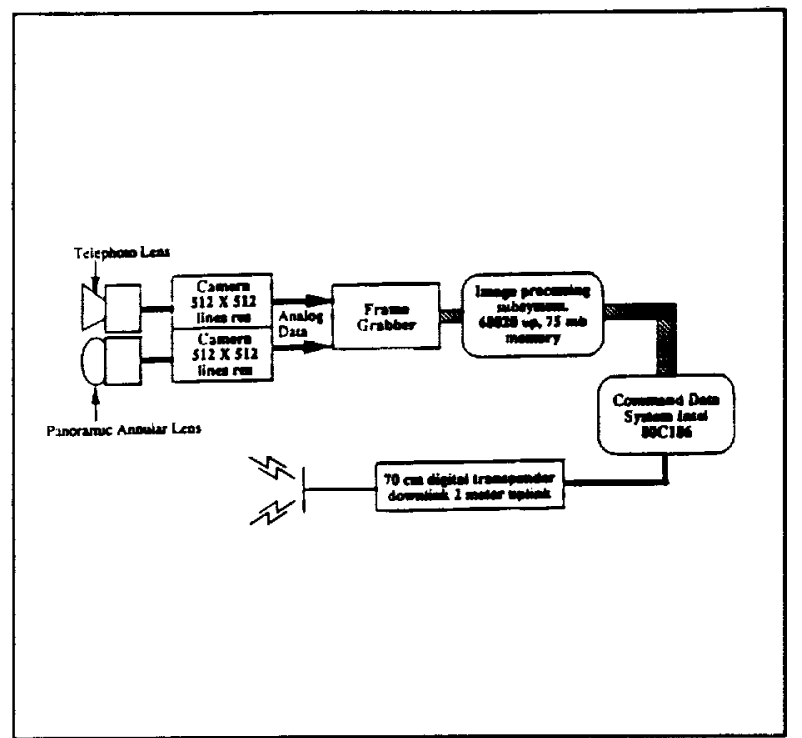

Figure 17. Schematic diagram of SEASIS.

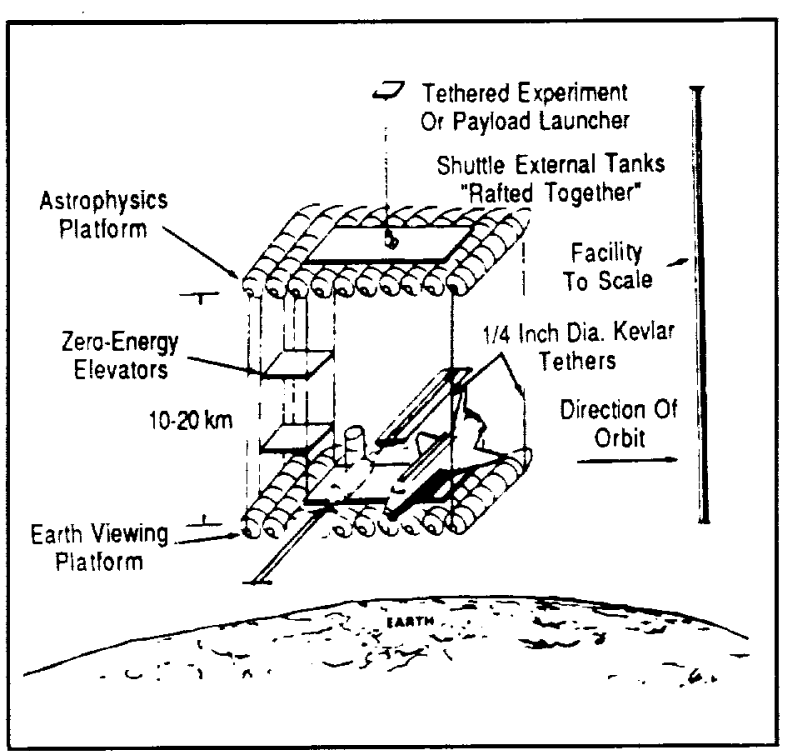

Figure 18. A space platform showing various tether applications.

variable/controlled gravity environment for materials processing, or to study long-term effects on humans. In the future, PAL systems may be used to establish accurate frames of reference for such space based construction. Figure 19, for example, shows a PAL image taken by the staff of Optechnology, Inc. while demonstrating a system for panoramically assisted rendezvous and capture (PARC) to Boeing and NASA. PARC is currently being considered for berthing operations aboard Space Station Freedom.

PAL systems may also be valuable for in-space inspection of spaceassembled structures, deployed structures, and manned systems. A typical monitoring sequence

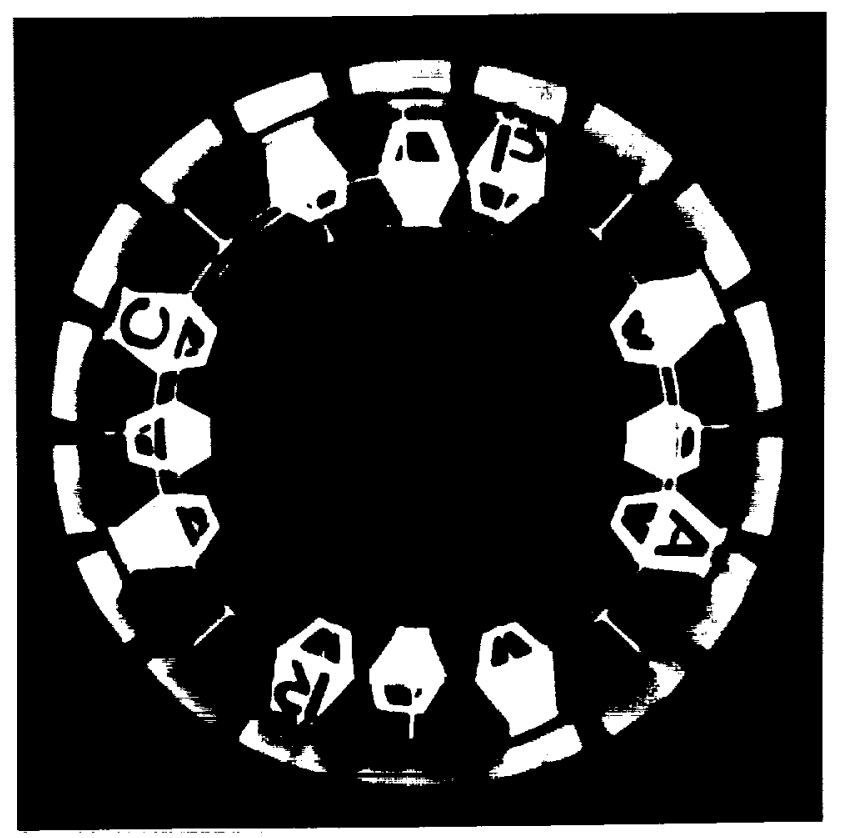

Figure 19. A PAL image taken from Optechnology's PARC system. may include characterization of defects or alignment of subsystems appropriate with mission requirements. The parameters to be monitored may include dimensional precision, and the stiffness or strength of truss members, 
joints, beams and structural supports. Inspections may be performed to look for cracks or deformation, externally-induced damage occurring as a result of impact from space debris or meteorites, or internally-induced damage such as fatigue or creep resulting from changes in frequency, stiffness, or temperature.

Other applications may include detection or characterization of flaws in composite materials, detection of inhomogeneities in metal castings, determination or verification of internal geometry, assessment of internal configuration and failure analysis support, detection of materials anomalies, determination of structural integrity, development of acceptance criteria for critical components, and impact damage tolerance studies.

A formidable challenge for the future will be to apply PAL systems in space to detect potential major and minor failures of structures and structural elements, leaks, and degradation of systems and subsystems over time and under conditions with which there is a very limited experience base. This challenge is magnified by the constraints imposed by the space environment such as operating in a vacuum and under micro-gravity conditions. Factors influencing the choice of instrumentation will include costs of development, installation, operation, portability, and transportability. The ease of operation, weight, size, sensitivity, reliability, power requirements, and the need for human intervention must also be considered.

\section{Personnel}

The following graduate students received direct financial support:

David Lehner (Ph.D. expected June, 1992 from UAH)

Wei Su (M.S. expected June, 1992 from UAH)

Joseph Puliparambil (Ph.D. expected June, 1992 from Marquette)

Other students at UAH and Marquette who contributed to the effort include Cheryl Bankston, Scott Caldwell, Sara Fair, Sally Gronner, Christelle Hendren, Patrick Meyer, Michael Popp, and Andrew Richter.

\section{Publications and Presentations}

Funding provided for this project helped support the following creative works:

Seminars:

1. Gilbert, J.A., Matthys, D.R., "Panoramic viewing systems," Sandia National Laboratories, Albuquerque, NM, February 4, 1991.

2. Gilbert, J.A. "Panoramic inspection and measurement, "Huntsville Electro-Optical Section and Working Group, Redstone Arsenel, Huntsville, Alabama, October 16, 1991. 
Presentations:

1. Gilbert, J.A., Matthys, D.R., Greguss, P., "Optical measurements through panoramic imaging systems," Proc. of the 1990 Int. Conf. on Hologram Interferometry \& Speckle Metrology, Baltimore, Maryland, November 4-7, 1990, pp. 164-171.

2. Gilbert, J.A., Matthys, D.R., Lehner, D.L., Hendren, C.M., "Panoramic imaging systems for nondestructive evaluation," Proc. of the Third Conference on Nondestructive Evaluation for Aerospace Requirements, Huntsville, Alabama, June 4-6, 1991.

3. Gilbert, J.A., Matthys, D.R., Hendren, C.M., "Displacement analysis of the interior walls of a pipe using panoramic holo-interferometry," Proc. of SPIE's 1991 International Symposium on Optical \& Optoelectronic Applied Science \& Engineering, San Diego, California, July 21-26, 1991.

4. Gilbert, J.A., Matthys, D.R., Lehner, D.L., "Moire measurements using a panoramic annular lens," Proc. of SPIE's 1991 International Symposium on Optical \& Optoelectronic Applied Science \& Engineering, San Diego, California, July 21-26, 1991.

5. Matthys, D.R., Gilbert, J.A., Puliparambil, J., "Endoscopic inspection using a panoramic annular lens," Proc. of SPIE's 1991 International Symposium on Optical \& Optoelectronic Applied Science \& Engineering, San Diego, California, July 21-26, 1991.

6. Gilbert, J.A., Bankston, C.D., "A "PARC" system for terminal docking," Proc. of the U.S. Automated Rendezvous and Capture Capabilities Review, Office of Space Flight, NASA Headquarters, Williamsburg, Virginia, November 19-21, 1991.

7. Gilbert, J.A., Fair, S.B., "A panoramic endoscope," Proc. of SPIE's Symposium on Optics, Electro-Optics, and Laser Applications in Science and Engineering, Los Angeles, CA, January 19-24, 1992.

Publication:

1. Matthys, D.R., Gilbert, J.A., Greguss, P., "Endoscopic measurement using radial metrology with digital correlation," Optical Engineering, 30(10): 1455-1460, 1991.

\section{Conclusions}

A new panoramic imaging system has been designed for visual inspection of aerospace components. It relies on a unique panoramic annular lens (PAL) which produces a flat annular image of the entire 360 degree surround of its optical axis. The most outstanding attributes of a PAL system are that there are no moving parts, the area surrounding the lens can be viewed simultaneously, and the depth of field extends from the surface of the PAL to infinity. The annular image may also be linearized for improved human viewing.

PAL systems are currently providing a new inspection capability for the SSME program and are expected to be applied for cavity inspection to verify the condition of space hardware after manufacturing, to perform inservice inspections following ground test engine firings, and to predict potential failure sites during refurbishment between shuttle flights. Current work to evaluate the potential for identifying and locating internal flaws, measuring the depth of surface 
cracks, comparing design contours to actual part contours, performing automated dimensional inspections, and imaging the relationship of the details in a complex assembly will be of tremendous importance to the space program.

With their unique imaging capabilities, PAL systems may also become an integral part of the Space Station Program and the manned expeditions projected by President Bush as part of the Space Exploration Initiative. As we look to the near-term future in space, they may be used for applications ranging from performing on-orbit nondestructive evaluation to guiding rovers over the lunar surface.

\section{Acknowledgements}

The authors would like to acknowledge the interest and support of several personnel at the Marshall Space Flight Center including Jack Lee, Director; Bill Lucas, former Director; Ann Whitaker, Ken Woodis, and Ron Beshears of the Materials and Processes Laboratory; John McCarty, Eric Hyde, Gary Lyles, and Jay Nichols of the Propulsion Laboratory; James Moses of the Research and Technology Office; and, Jonathan Campbell of the Space Sciences Laboratory who first entertained our proposal to "Change The World's Perspective." 